\title{
Sagittal alignment changes and postoperative complications after adult spinal deformity surgery in patients with Parkinson's disease: A multi- institutional retrospective study
}

\section{Atsuyuki Kawabata}

Tokyo Medical and Dental University

Toshitaka Yoshii ( $\square$ yoshii.orth@tmd.ac.jp )

Tokyo Medical and Dental University

Kenichiro Sakai

Saiseikai Kawaguchi General Hospital

Takashi Hirai

Tokyo Medical and Dental University

Masato Yuasa

Tokyo Medical and Dental University

Hiroyuki Inose

Tokyo Medical and Dental University

\section{Yu Matsukura}

Saiseikai Kawaguchi General Hospital

Masaki Tomori

Saiseikai Kawaguchi General Hospital

Ichiro Torigoe

Saiseikai Kawaguchi General Hospital

Kazuo Kusano

Kudanzaka Hospital

Kazuyuki Otani

Kudanzaka Hospital

Yoshiyasu Arai

Saiseikai Kawaguchi General Hospital

Shigeo Shindo

Kudanzaka Hospital

\section{Atsushi Okawa}

Tokyo Medical and Dental University 
Keywords: adult spinal deformity, Parkinson's disease, postoperative complications, sagittal alignment, sagittal vertical axis, radiographic parameter

Posted Date: February 11th, 2020

DOI: https://doi.org/10.21203/rs.2.23127/v1

License: (a) (i) This work is licensed under a Creative Commons Attribution 4.0 International License. Read Full License

Version of Record: A version of this preprint was published at BMC Musculoskeletal Disorders on April 16th, 2021. See the published version at https://doi.org/10.1186/s12891-021-04233-5. 


\section{Abstract}

Background: Parkinson's disease (PD) has been reported to increase the risk of postoperative complications in patients with adult spinal deformity (ASD). However, those reports are limited, and few have made direct comparisons with patients who do not have PD.

Methods: We retrospectively reviewed all surgically treated patients with ASD and at least 2 years of followup. Among them, 27 had PD (PD(+) group). Clinical data were collected on early and late postoperative complications and revision surgery. Radiographic parameters were evaluated before and immediately after surgery and at final follow-up, including sagittal vertical axis (SVA), thoracic kyphosis, lumbar lordosis, sacral slope, and pelvic tilt. From the same database, we also retrieved 206 controls without PD (PD(-) group) matched for age, sex, and body mass index.

Results: For early complications, the PD(+) group showed a higher rate of delirium than the $\mathrm{PD}(-)$ group. Deep vein thrombosis and pulmonary embolism rates tended to be higher in the $\mathrm{PD}(+)$ group. For late complications, the rate of pseudarthrosis was significantly higher in the PD $(+)$ group. Rates of rod failure and revision surgery due to mechanical complications also tended to be higher, but not significantly, in the $\mathrm{PD}(+)$ group $(p=0.17, p=0.13$, respectively). SVA at final follow-up and loss of correction in SVA were significantly higher in the $\mathrm{PD}(+)$ group.

Conclusion: Extra attention should be paid to perioperative complications, especially delirium and thrombosis, in PD patients undergoing surgery for ASD. Furthermore, loss of correction and rate of revision surgery due to mechanical complications were higher in these patients.

\section{Background}

Parkinson's disease (PD) is a neurodegenerative disorder, the main symptoms of which are rigidity, bradykinesia, and gait disorder. With population aging, the number of patients with this age-related disorder is increasing[1]. In severe cases, PD causes postural disorder, such as anterocollis, Pisa syndrome, and camptocormia [2-5]. Reports have shown that these postural abnormalities increase susceptibility to rigid spinal deformities $[2,3]$ and that patients with PD develop adult spinal deformity (ASD) more frequently than the general population in the same age groups [6].

Recent studies have shown that ASD negatively affects health-related quality of life $[7,8]$. Surgical treatment has been shown to provide better health-related quality of life outcomes than non-surgical treatment, especially in patients with severe deformity [9]. However, high complication rates have been reported with revision rates of up to $47 \%$. Surgical complications are generally divided into perioperative and late complications. Perioperative complications include epidural hematoma, deep vein thrombosis (DVT), and pulmonary embolism (PE), which are usually seen during or soon after surgery. Late complications, such as junctional kyphosis, rod fracture, and pseudarthrosis, typically occur more than 1 month after surgery and are mainly caused by continuous mechanical stress. 
Generally, PD patients are presumed to have a higher risk of surgical complications, especially mechanical complications due to postural instability, a higher risk of falls, and lower bone quality [10-13] However, to date, only a few small case series have investigated complications after surgery for ASD in PD patients [14-17]. In addition, risk factors for complications and revision surgery in PD patients have not yet been confirmed. Therefore, we conducted this multicenter study to investigate 234 surgically treated ASD patients with at least 2 years' follow-up. We compared surgical outcomes and radiographic parameters of PD patients with those of non-PD patients matched for age, sex, and body mass index (BMI). We also investigated risk factors for revision surgery in PD patients.

\section{Methods}

This retrospective observational study follows the Strengthening the Reporting of Observational studies in Epidemiology (STROBE) guidelines. We reviewed 233 ASD patients treated surgically between January, 2009, through December, 2016, at our hospital and related institutions. Institutional review board approval was obtained at each site for the patient enrollment and data collection protocols. Inclusion criteria were age 21 years or older at the time of surgery, follow-up period of at least 2 years, and surgery including posterior instrumentation of 4 spinal levels with sufficient radiographic data. A total of 27 patients with PD were identified. Data on mean PD duration and Hoehn and Yahr (HY) stage were collected. A control group of 206 non-PD patients was retrieved from the same database.

Etiologies included degenerative kyphosis/kyphoscoliosis, post lumbar surgery, and previous vertebral fracture. Operative time and intraoperative blood loss were recorded. Perioperative complications were defined as complications that occurred during and within 1 month after surgery, including neurological disorder, implant failure, DVT, PE, cerebrovascular disease, respiratory disorder, cardiovascular disorder, delirium, surgical site infection, and spinal epidural hematoma. The number of revision surgeries due to perioperative complications was also noted. Mechanical complications were defined as proximal junctional kyphosis (PJK), distal junctional kyphosis (DJK), pseudarthrosis, rod breakage, and vertebral fracture with or without revision surgery. PJK was defined as kyphosis $>10^{\circ}$ between the upperinstrumented vertebra (UIV) and two-level proximal vertebrae. Mechanical failure was defined as mechanical complications requiring revision surgery.

Demographic data were collected including age, sex, BMI, medical comorbidities, and bone mineral density (BMD) of the femoral neck. Measurements on radiographs included SVA, thoracic kyphosis (T4-T10), L1S1 lumbar lordosis (LL), sacral slope, pelvic incidence (PI), and pelvic tilt. These parameters were evaluated before and at 4 weeks after surgery in the standing position. X-ray images were also evaluated at the final follow-up. A three-column osteotomy was defined as a procedure using pedicle subtraction osteotomy or vertebral column resection. The radiographic parameter of $-10^{\circ}<\mathrm{PI}-\mathrm{LL}<10^{\circ}$ was defined as ideal alignment based on the SRS-Schwab ASD classification.

Statistical analysis was performed using IBM SPSS Statistics for Macintosh, Version 25.0 (Released 2017 IBM Corp, Armonk, NY). We divided all patients into two groups: patients with PD (PD $(+)$ group) and patients without $\mathrm{PD}(\mathrm{PD}(-)$ group. We used a paired t-test or chi-squared test to compare the $\mathrm{PD}(+)$ and the 
$\mathrm{PD}(-)$ groups. Also, t-tests were used to compare the means of continuous variables and chi-square tests to compare the proportions of categorical variables between the groups. A p-value $<0.05$ was considered statistically significant. Missing values were imputed using the last observation carried forward method.

\section{Results}

Table 1 shows the patient characteristics of the $\mathrm{PD}(+)$ group and the $\mathrm{PD}(-)$ group. There was no significant difference between the two groups in age, sex, BMI, or BMD. The number of fixed levels was significantly higher in the $\mathrm{PD}(+)$ group than in the $\mathrm{PD}(-)$ group $(9.3 \pm 2.6$ vs $7.8 \pm 1.9, p=0.006)$ and the rates of threecolumn osteotomy tended to be higher in the $\mathrm{PD}(+)$ group $(63.0 \%$ vs $48.5 \%, p=0.14)$. For preoperative radiographic parameters, SVA was significantly higher in the $\mathrm{PD}(+)$ group than in the $\mathrm{PD}(-)$ group $(196.0 \pm$ $63.8 \mathrm{~mm}$ vs $135.4 \pm 69.9 \mathrm{~mm}, p<0.001)$. Other parameters were not significantly different between these groups. In terms of etiology, ASD caused by previous vertebral fracture was significantly higher in the PD(+) group than in the $\mathrm{PD}(-)$ group (previous vertebral fracture/degenerative/post lumbar surgery: $38.5 \% / 53.8 \% / 7.7 \%$ vs $10.1 \% / 64.7 \% / 25.1 \%, p=0.005)$.

Table 1. Demographic and operative characteristics of the PD group and non-PD group. 


\begin{tabular}{|c|c|c|c|}
\hline & PD & Non-PD & $P$-value \\
\hline No. of cases & 27 & 206 & - \\
\hline Age at surgery (years) & $70.6 \pm 6.3$ & $72.5 \pm 8.5$ & 0.18 \\
\hline Sex (male/female: cases) & $5 / 22$ & $32 / 174$ & 0.58 \\
\hline BMI & $22.8 \pm 4.2$ & $23.2 \pm 3.7$ & 0.59 \\
\hline BMD (t-score) & $-1.900 \pm 1.249$ & $-1.863 \pm 0.924$ & 0.93 \\
\hline Number of fixed levels & $9.3 \pm 2.6$ & $7.8 \pm 1.9$ & 0.006 \\
\hline $3 C O$ (yes/no) & $17 / 10$ & $100 / 106$ & 0.14 \\
\hline Etiologies(post fracture/degenerative/previous lumbar fusion) & $10 / 15 / 2$ & $21 / 133 / 52$ & 0.001 \\
\hline Pre SVA & $196.0 \pm 63.7$ & $135.4 \pm 69.9$ & $<0.001$ \\
\hline Pre LL & $2.1 \pm 19.3$ & $3.5 \pm 20.5$ & 0.75 \\
\hline Pre TLK & $18.1 \pm 16.0$ & $19.2 \pm 17.0$ & 0.28 \\
\hline Pre TK & $24.1 \pm 15.4$ & $27.4 \pm 20.0$ & 0.37 \\
\hline Pre SS & $17.1 \pm 8.0$ & $14.7 \pm 11.4$ & 0.34 \\
\hline PI & $53.6 \pm 9.6$ & $49.8 \pm 9.1$ & 0.088 \\
\hline \multicolumn{4}{|c|}{ No, number; PD, Parkinson disease; BMI, bone mass index; BMD, bone mineral density; } \\
\hline TK, Thoracic kyphosis; SS, Sacral slope; PI, pelvic incidence & & & \\
\hline
\end{tabular}

Table 2 shows surgical invasiveness and postoperative complications in each group. There were no significant differences in operative time and intraoperative blood loss between the two groups. For early complications, the PD(+) group showed a higher rate of delirium than the PD(-) group. In the PD(+) group, rates of DVT and PE tended to be higher (DVT: $14.8 \%$ vs $6.8 \%, p=0.12 ; \mathrm{PE}: 3.7 \%$ vs $0.5 \%, p=0.081$ ). There were no significant differences between the groups in the rates of other complications, including neurological deficits, implant failure, cerebrovascular disorder, respiratory disorder, cardiovascular disorder, and surgical site infection. There was no significant difference in the rate of revision surgery due to early complications. For late complications, the rate of pseudarthrosis was significantly higher in the $\mathrm{PD}(+)$ group (15.3\% vs 3.9\%; $p=0.013)$. Rates of rod failure and revision surgery due to mechanical 
complications tended to be higher, but not significantly, in the PD(+) group (rod failure: $25.9 \%$ vs $13.1 \%, p=$ 0.174 ; revision surgery: $33.3 \%$ vs $18.0 \%, p=0.13$ ).

Table 2. Postoperative complications of the PD (+) group and the PD (-) group. 


\begin{tabular}{llll}
\hline & PD (+) & PD (-) & $P$-value \\
\hline No. of cases & 27 & 206 & - \\
& & & \\
Surgical time (min) & $490 \pm 154$ & $454 \pm 113$ & 0.14 \\
Blood loss (g) & $2069 \pm 1336$ & $1984 \pm 1422$ & 0.28
\end{tabular}

\section{Early complications}

$\begin{array}{llll}\text { Neurological complications (yes/no) } & 3 / 24(11.1 \%) & 23 / 183(11.2 \%) & 0.96 \\ \text { Implant failure } & 2 / 25(7.4 \%) & 20 / 186(9.7 \%) & 0.74 \\ \text { DVT } & 4 / 23(14.8 \%) & 14 / 192(6.8 \%) & 0.12 \\ \text { PE } & 1 / 26(3.7 \%) & 1 / 205(0.5 \%) & 0.081 \\ \text { Cerebrovascular disorder } & 0 / 27(0 \%) & 2 / 204(1.0 \%) & 0.61 \\ \text { Respiratory disorder } & 1 / 26(3.7 \%) & 7 / 199(3.4 \%) & 0.91 \\ \text { Cardiovascular disorder } & 0 / 27(0 \%) & 6 / 200(2.9 \%) & 0.38 \\ \text { Delirium } & 7 / 20(25.9 \%) & 15 / 191(7.3 \%) & 0.001 \\ \text { Surgical site infection } & 1 / 26(3.7 \%) & 6 / 200(2.9 \%) & 0.79 \\ \text { Spinal epidural hematoma } & 0 / 27(0 \%) & 14 / 192(6.8 \%) & 0.17 \\ \text { Revision } & 2 / 25(7.4 \%) & 30 / 176(14.6 \%) & 0.34\end{array}$

Late complications

\begin{tabular}{llll} 
Mechanical complication & $15 / 12(55.6 \%)$ & $99 / 107(48.1 \%)$ & 0.59 \\
Rod failure & $7 / 20(25.9 \%)$ & $27 / 179(13.1 \%)$ & 0.17 \\
PJK & $7 / 20(25.9 \%)$ & $57 / 149(27.7 \%)$ & 0.91 \\
DJK & $3 / 24(11.1 \%)$ & $15 / 191(7.3 \%)$ & 0.95 \\
Pseudoarthrosis & $5 / 22(18.5 \%)$ & $8 / 198(3.9 \%)$ & 0.013 \\
Vertebral fracture & $7 / 20(25.9 \%)$ & $43 / 163(20.9 \%)$ & 0.81 \\
Revision & $9 / 18(33.3 \%)$ & $37 / 169(18.0 \%)$ & 0.13 \\
\hline
\end{tabular}

No, number; PD, Parkinson disease; DVT, deep vein thrombosis; PE, pulmonary embolism; PJK, proximal junction kyphosis; DJK, distal junction kyphosis 
Table 3 summarizes changes in radiographic parameters postoperatively and at final follow-up. Postoperative SVA was similar between the $\mathrm{PD}(+)$ and $\mathrm{PD}(-)$ groups, although preoperative SVA was much higher in the $\mathrm{PD}(+)$ group. The change in SVA between pre- and postoperative radiographs was significantly higher in the $\mathrm{PD}(+)$ group $(-142.5 \pm 82.0 \mathrm{~mm}$ vs $-94.6 \pm 69.7 \mathrm{~mm}, p=0.014)$. In addition, SVA at the final follow-up tended to be higher in the $\mathrm{PD}(+)$ group $(p=0.062)$. Loss of correction in SVA also tended to be higher in the $\mathrm{PD}(+)$ group $(p=0.11)$. No significant differences were found in other radiographic parameters (Fig. 1), including the proposed ideal alignment target of $\mathrm{PI}-\mathrm{LL}<10$.

Table 3. Postoperative radiographic parameters of the PD group and non-PD group.

\begin{tabular}{llll}
\hline & PD (+) & PD (-) & $P$-value \\
\hline No. of cases & 27 & 207 & - \\
Post SVA & $53.5 \pm 41.6$ & $40.0 \pm 46.2$ & 0.17 \\
Change of SVA (post-pre) & $-142.5 \pm 82.0$ & $-94.6 \pm 69.7$ & 0.003 \\
Final SVA & $89.2 \pm 68.1$ & $59.9 \pm 52.2$ & 0.062 \\
SVA correction loss & $35.7 \pm 62.1$ & $19.2 \pm 43.4$ & 0.11 \\
Post LL & $41.4 \pm 10.8$ & $39.7 \pm 11.3$ & 0.64 \\
Final LL & $38.6 \pm 12.3$ & $36.4 \pm 12.5$ & 0.74 \\
LL correction loss & $-0.64 \pm 20.9$ & $-0.20 \pm 21.3$ & 0.93 \\
Post TK & $37.1 \pm 12.9$ & $36.5 \pm 14.5$ & 0.85 \\
Final TK & $44.3 \pm 14.8$ & $43.0 \pm 16.5$ & 0.70 \\
TK correction loss & $7.3 \pm 7.1$ & $6.5 \pm 10.6$ & 0.87 \\
Post SS & $27.1 \pm 6.6$ & $26.2 \pm 8.6$ & 0.56 \\
Final SS & $27.4 \pm 9.6$ & $24.7 \pm 17.3$ & 0.48 \\
SS correction loss & $-0.3 \pm 6.4$ & $1.7 \pm 16.8$ & 0.58 \\
$-10<$ post PI-LL<10 (yes/no) & $12 / 15$ & $108 / 99$ & 0.83 \\
\hline
\end{tabular}

No, number; PD, Parkinson disease; SVA, sagittal vertical axis; LL, lumbar lordosis;

TK, Thoracic kyphosis; SS, Sacral slope; PI, pelvic incidence

Table 4 shows the preoperative demographics, postoperative radiographic parameters, and surgical characteristics of the PD group comparing those who underwent revision surgery (revision subgroup) with those who did not (non-revision subgroup). No significant difference was found in the radiographic parameters between these two groups. For preoperative demographics, the duration of PD was significantly higher in the revision subgroup ( $87.0 \pm 56.9$ months vs $32.5 \pm 48.0$ months, $p=0.037)$. HY 
stage was higher in the revision subgroup, although this was not significantly different $(2.8 \pm 1.2$ vs $2.2 \pm$ $1.3, p=0.29)$.

Table 4. Postoperative radiographic parameters and demographics of revision group and non-revision group in the PD group.

\begin{tabular}{|c|c|c|c|}
\hline & revision $(+)$ & revision (-) & $P$-value \\
\hline No. of cases & 9 & 18 & - \\
\hline Pre SVA & $187.0 \pm 62.3$ & $193.4 \pm 63.6$ & 0.84 \\
\hline Post SVA & $60.2 \pm 27.4$ & $57.7 \pm 39.2$ & 0.87 \\
\hline Pre LL & $-3.5 \pm 27.3$ & $4.9 \pm 16.3$ & 0.51 \\
\hline Post LL & $33.7 \pm 13.4$ & $44.6 \pm 8.6$ & 0.11 \\
\hline Pre TLK & $9.3 \pm 6.9$ & $11.2 \pm 12.5$ & 0.26 \\
\hline Post TLK & $11.4 \pm 11.4$ & $0.2 \pm 11.6$ & 0.074 \\
\hline Pre TK & $15.0 \pm 16.0$ & $26.3 \pm 13.7$ & 0.17 \\
\hline Post TK & $30.0 \pm 18.5$ & $40.1 \pm 9.8$ & 0.25 \\
\hline Pre SS & $16.2 \pm 11.8$ & $17.0 \pm 6.5$ & 0.87 \\
\hline Post SS & $24.3 \pm 7.8$ & $28.5 \pm 6.2$ & 0.28 \\
\hline PI & $51.7 \pm 8.7$ & $54.6 \pm 10.4$ & 0.53 \\
\hline Age at surgery & $68.5 \pm 3.7$ & $71.6 \pm 6.5$ & 0.19 \\
\hline BMI & $21.9 \pm 3.7$ & $23.8 \pm 4.3$ & 0.33 \\
\hline The number of level fixed & $9.0 \pm 2.5$ & $10.2 \pm 2.5$ & 0.35 \\
\hline Disease duration of PD & $87.0 \pm 56.9$ & $32.5 \pm 48.0$ & 0.037 \\
\hline Hoehn and Yahr stage & $2.8 \pm 1.2$ & $2.2 \pm 1.3$ & 0.29 \\
\hline BMD (T score) & & $-1.8 \pm 1.2$ & 0.81 \\
\hline
\end{tabular}

No, number; PD, Parkinson disease; SVA, sagittal vertical axis; LL, lumbar lordosis;

TLK, Thoracolumbar kyphosis; TK, Thoracic kyphosis; SS, Sacral slope;

PI, pelvic incidence; BMI, bone mass index, BMD, bone mineral density 
PD patients have been shown to have more postoperative surgical complications due to various musculoskeletal problems. Studies have investigated high rates of perioperative and postoperative complications following hip and knee surgery in PD patients using a nationwide inpatient database [18, 19]. Furthermore, other surgical studies on the PD population have suggested high rates of postoperative medical complications such as pneumonia, delirium, and sepsis [5]. However, few case series have examined the relationship between PD and complications following corrective surgery for ASD.

Furthermore, few studies have focused on postoperative loss of correction of radiographic parameters in PD patients. Thus, we retrospectively analyzed both postoperative complications and radiographic parameters in this multicenter database of ASD patients and compared PD patients with non-PD patients matched for age, sex, and BMI with at least 2-years' follow-up.

The results of this study demonstrated that the rate of delirium was significantly higher, and the rates of PE and DVT tended to be higher in the PD(+) group. In addition, Watanabe et al. previously reported that postoperative delirium was more common in patients with PD (23.1\%) than in the controls (3.4\%) [20]. This higher rate of delirium is possibly due to the use of antidopaminergic drugs and neurodegeneration in PD patients. Although delirium is a reversible condition, it is necessary to avoid additional complications due to falls or movement beyond the limits of restriction. For thrombotic events, PE is known to be a possible adverse reaction to antidopaminergic drugs, such as levodopa [21]. Yamane et al. reported a higher incidence (20\%) of DVT in PD patients with a postural abnormality [22]. Since the correction surgery for ASD itself has a high risk for PE due to its long surgical time and large blood loss, it is assumed that the risk of PE of this surgery in PD patients is very high. In fact, in this study, the incidence of PE in PD patients was 7 times higher and that of DVT was about 2 times higher than in non-PD patients. Surgeons should thus consider PE in patients who develop chest pain and dyspnea after surgery.

In this study, we found significantly higher preoperative SVA and larger correction of SVA in the PD(+) group. We also found higher SVA at the final follow-up and higher loss of correction in SVA, even though longer fusion was performed in the $\mathrm{PD}(+)$ group. This finding suggests that the deformities in the PD(+) group were mostly flexible and largely corrected by the surgery. However, the corrected alignment could not be well maintained in this group, suggesting that a larger correction can lead to a higher rate of loss of correction. In addition, the progressive stooping posture of PD patients can pose a risk for loss of correction of global sagittal alignment, and this may be one of the causes for the poorer outcome in PD patients. Kawaguchi et al. reported that longer fusion until T4 yielded a good clinical outcome in a PD patient after corrective surgery for L1 to S1 was unsuccessful [23]. Watanabe et al. reported on 8 surgically treated ASD patients with PD who demonstrated poor clinical outcomes with a high non-union rate and adjacent segment disease [24]. Thus, it is crucial to consider possible prevention strategies, including longer fusion.

Previous studies reported that PD patients have a higher rate of osteoporotic vertebral fracture and lower BMD $[1,20]$. However, we found no significant difference in BMD in this study even though the rates of previous vertebral fractures were significantly higher in the PD group. We assumed that the postural abnormality in the PD(+) group added greater burden on the vertebral body than on that of a healthy person and resulted in vertebral fracture. Thus, caution should be exercised when considering the risk of 
PJK type fracture in PD patients. Yagi et al. reported that teriparatide treatment started immediately after surgery resulted in a decrease in rates of PJK-type bone fracture [25]. If BMD is low, then administration of osteoporotic drugs such as teriparatide could be a viable option to decrease the risk of complications, as suggested in the previous study.

This study showed that the revision rate due to mechanical complications was $33.3 \%$, which was almost two times higher in the $\mathrm{PD}(+)$ group than in the $\mathrm{PD}(-)$ group. Similar to our study, Sheu et al. investigated 66 PD patients who underwent thoracolumbar or lumbar instrumented surgery due to degeneration or deformity; $29 \%$ of them needed revision surgery due to mechanical complications [13]. Bouyer et al. reported a high revision rate of $42 \%$ in 48 ASD patients with PD, $89 \%$ of which were due to mechanical complications [16]. In terms of each complication, PJK has been reported to be significantly higher in PD patients. In contrast, a history of PD had no significant impact on the PJK rate in this study. Instead, rates of pseudarthrosis and rod failure were higher in the $\mathrm{PD}(+)$ group. This was probably because a longer fusion was performed in the $\mathrm{PD}(+)$ group, possibly as a result of the surgeon's decision considering the severe deformity with higher SVA. Long-segment fixation may lower the risk of PJK but the stress on the rod and the mobile segment of the spine can be higher and may have caused rod fracture and pseudarthrosis.

Several studies have investigated risk factors for revision surgery in PD patients. Schroeder et al. reported that an $\mathrm{HY}$ stage $>2$, diabetes mellitus, treatment for osteoporosis, and a combined anterior and posterior approach were risk factors for revision surgery in 94 lumbar spine surgeries [12]. According to Sheu et al., $\mathrm{HY}$ stage $>2$, cancer history, osteoporosis, and three column osteotomy were risk factors for revision surgery [13]. In our study, PD duration was significantly longer in the revision subgroup, even though they were younger. HY stage can be affected by symptoms of sagittal malalignment and imbalance in ASD patients, and thus the stage itself may not reflect the exact severity of PD in ASD patients. However, the HY stage also tended to be higher in the revision subgroup. As the previous study indicated, poorly controlled PD negatively impacts the incidence of revision surgery. Thus, it is vital to consider duration and severity of PD when performing surgical treatment in ASD patients.

There are several limitations to this study. First, there was a selection bias in which the surgeon could change their choice of a surgical procedure because of the patient's PD status. Second, our study was retrospective in nature. Studies with more appropriate study designs and a larger sample size are needed. Despite these limitations, our study demonstrated surgical outcomes in patients with PD compared with patients without PD using a large database with a sufficient follow-up period.

\section{Conclusion}

In patients with PD, postoperative delirium was common and the rate of PE and DVT tended to be higher. Late complications included rod fracture, pseudarthrosis, and revision surgery, which were higher in the $\mathrm{PD}(+)$ group. The rate of revision surgery due to mechanical complications was about two times that of the $\mathrm{PD}(-)$ group. In the PD (+) group, SVA was significantly larger before surgery and at final follow-up, as was loss of correction in SVA, suggesting that PD-specific postural abnormalities are involved in sagittal 
parameter deterioration. PD duration was significantly higher in the PD patients who underwent revision surgery than in those who did not.

\section{List Of Abbreviations}

ASD: adult spinal deformity; BMI: body mass index; BMD: bone mineral density; DVT: deep vein thrombosis; DJK: distal junctional kyphosis; HY: Hoehn and Yahr; LL: L1-S1 lumbar lordosis; PD:

Parkinson's disease; PI: pelvic incidence; PJK: proximal junctional kyphosis; PE: pulmonary embolism; T4T10: thoracic kyphosis; UIV: upper-instrumented vertebra

\section{Declarations}

\section{Ethics approval and consent to participate}

The study was approved by the ethics committee of all institutions involved. Informed consent was waivered by the above ethics committee as the present retrospective cohort study involved already existing data and records at the time of investigation, and did not retain personal identifiers of the gathered information.

The ethical committee, Tokyo Medical and Dental University; M2017-115

The ethical committee, Saiseikai Kawaguchi General Hospital; 29-2

The ethical committee, Kudanzaka Hospital; 2019-5

\section{Consent for Publication}

Not applicable

\section{Availability of data and materials}

The datasets generated during and/or analysed during the current study are available from the corresponding author on reasonable request.

\section{Competing interests}

Not applicable

\section{Funding}

Not applicable

\section{Authors' contributions}

AK analysed the data and wrote the original draft. TY conceived of, reviewed, and edited the paper. KS, TH, MY, HI, YM, MT, IT, and KK acquired the data. KO performed the investigation. YA validated the paper. YA, 
SS, and AO supervised the researched. All authors contributed to the writing of the final manuscript. All authors approved the manuscript to be published and agree to be accountable for all aspects of the work in ensuring that questions related to the accuracy or integrity of any part of the work are appropriately investigated and resolved.

\section{Acknowledgements}

Not applicable.

\section{References}

1. de Lau LM, Breteler MM. Epidemiology of Parkinson's disease. Lancet Neurol. 2006;5:525-535. https://doi.org/10.1016/S1474-4422(06)70471-9

2. Doherty KM, Davagnanam I, Molloy S, Silveira-Moriyama L, Lees AJ. Pisa syndrome in Parkinson's disease: a mobile or fixed deformity? J Neurol Neurosurg Psychiatry. 2013;84:1400-1403. https://doi.org/10.1136/jnnp-2012-304700

3. Ye X, Lou D, Ding X, Xie C, Gao J, Lou Y, et al. A clinical study of the coronal plane deformity in Parkinson disease. Eur Spine J. 2017;26:1862-1870. https://doi.org/10.1007/s00586-017-5018-6

4. Alwardat M, Schirinzi T, Di Lazzaro G, Franco D, Sinibaldi Salimei P, Mercuri NB, et al. The effect of postural deformities on back function and pain in patients with Parkinson's disease.

NeuroRehabilitation. 2019;44:419-424. https://doi.org/10.3233/NRE-182637

5. Protopsaltis TS, Boniello AJ, Schwab FJ. Management of spinal deformity in adult patients with neuromuscular disease. J Am Acad Orthop Surg. 2016;24:634-644. https://doi.org/10.5435/JAAOS-D$15-00421$

6. Marsden CD, Duvoisin R. Scoliosis and Parkinson's disease. Arch Neurol. 1980;37:253-254. https://doi.org/10.1001/archneur.1980.00500530091025

7. Schwab FJ, Blondel B, Bess S, Hostin R, Shaffrey Cl, Smith JS, et al. Radiographical spinopelvic parameters and disability in the setting of adult spinal deformity: A prospective multicenter analysis. Spine (Phila Pa 1976). 2013;38:E803-812. https://doi.org/10.1097/BRS.0b013e318292b7b9

8. Scheer JK, Tang JA, Smith JS, Klineberg E, Hart RA, Mundis GM, et al. Reoperation rates and impact on outcome in a large, prospective, multicenter, adult spinal deformity database: Clinical article. J Neurosurg Spine. 2013;19:464-470.https://doi.org/10.3171/2013.7.SPINE12901

9. Acaroglu E, Yavuz AC, Guler UO, Yuksel S, Yavuz Y, Domingo-Sabat M, et al. A decision analysis to identify the ideal treatment for adult spinal deformity: Is surgery better than non-surgical treatment in improving health-related quality of life and decreasing the disease burden? Eur Spine J. 2016;25:23902400. https://doi.org/10.1007/s00586-016-4413-8

10. Baker JF, McClelland S, Line BG, Smith JS, Hart RA, Ames CP, et al. In- hospital complications and resource utilization following lumbar spine surgery in patients with Parkinson disease: Evaluation of the National Inpatient Sample database. World Neurosurg. 2017;106:470-476.

https://doi.org/10.1016/j.wneu.2017.07.006

Page 14/17 
11. Kimura H, Fujibayashi S, Otsuki B, Takemoto M, Shikata J, Odate S, et al. Lumbar spinal surgery in patients with Parkinson disease: A multicenter retrospective study. Clin Spine Surg. 2017;30:E809E818. https://doi.org/10.1097/BSD.0000000000000455

12. Schroeder JE, Hughes A, Sama A, Weinstein J, Kaplan L, Cammisa FP, et al. Lumbar spine surgery in patients with Parkinson disease. J Bone Joint Surg Am. 2015;97:1661-1666. https://doi.org/10.2106/JBJS.N.01049

13. Sheu H, Liao JC, Lin YC. The fate of thoracolumbar surgeries in patients with Parkinson's disease, and analysis of risk factors for revision surgeries. BMC Musculoskelet Disord. 2019;20:106. https://doi.org/10.1186/s12891-019-2481-8

14. Bourghli A, Guérin P, Vital JM, Aurouer N, Luc S, Gille 0 , et al. Posterior spinal fusion from T2 to the sacrum for the management of major deformities in patients with Parkinson disease: a retrospective review with analysis of complications. J Spinal Disord Tech. 2012;25:E53-60. https://doi.org/10.1097/BSD.0b013e3182496670

15. Babat LB, McLain RF, Bingaman W, Kalfas I, Young P, Rufo-Smith C. Spinal surgery in patients with Parkinson's disease: construct failure and progressive deformity. Spine (Phila Pa 1976). 2004;29:20062012. https://doi.org/10.1097/01.brs.0000138306.02425.21

16. Bouyer B, Scemama C, Roussouly P, Laouissat F, Obeid I, Boissière L, et al. Evolution and complications after surgery for spine deformation in patients with Parkinson's disease. Orthop Traumatol Surg Res. 2017;103:517-522. https://doi.org/10.1016/j.otsr.2016.12.024

17. Koller H, Acosta F, Zenner J, Ferraris L, Hitzl W, Meier O, et al. Spinal surgery in patients with Parkinson's disease: experiences with the challenges posed by sagittal imbalance and the Parkinson's spine. Eur Spine J. 2010;19:1785-1794. https://doi.org/10.1007/s00586-010-1405-y

18. Newman JM, Sodhi N, Wilhelm AB, Khlopas A, Klika AK, Naziri Q, et al. Parkinson's disease increases the risk of perioperative complications after total knee arthroplasty: a nationwide database study. Knee Surg Sports Traumatol Arthrosc. 2019;27:2189-2195. https://doi.org/10.1007/s00167-018-4970y

19. Min H, Lin H, Chen G. Effect of Parkinson's disease on primary total joint arthroplasty outcomes: A meta-analysis of matched control studies. Int J Surg. 2019;71:124-131. https://doi.org/10.1016/j.ijsu.2019.09.013

20. Watanabe K, Katsumi K, Ohashi M, Shibuya Y, Izumi T, Hirano T, et al. Surgical outcomes of spinal fusion for osteoporotic thoracolumbar vertebral fractures in patients with Parkinson's disease: what is the impact of Parkinson's disease on surgical outcome? BMC Musculoskelet Disord. 2019;20:103. https://doi.org/10.1186/s12891-019-2473-8

21. Hung SC, Tai CT. Parkinson's disease with recurrent pulmonary embolism. Zhonghua Yi Xue Za Zhi (Taipei). 2000;63:487-491

22. Yamane K, Kimura F, Unoda K, Hosokawa T, Hirose T, Tani H, et al. Postural abnormality as a risk marker for leg deep venous thrombosis in Parkinson's disease. PLoS One. 2013; doi: 10.1371/journal.pone.0066984 
23. Watanabe K, Hirano T, Katsumi K, Ohashi M, Shoji H, Hasegawa K, et al. Characteristics of spinopelvic alignment in Parkinson's disease: Comparison with adult spinal deformity. J Orthop Sci. 2017;22:1621. https://doi.org/10.1016/j.jos.2016.09.013

24. Watanabe K, Homma T, Yamazaki A. Surgical outcomes of spinal fusion surgery for lumbar degenerative scoliosis in patients with Parkinson's disease: Reconstruction failure and progressive deformity. J Spine Res. 2011;2:1911-1920 (in Japanese)

25. Yagi M, Ohne H, Konomi T, Fujiyoshi K, Kaneko S, Komiyama T, et al. Teriparatide improves volumetric bone mineral density and fine bone structure in the UIV+1 vertebra, and reduces bone failure type PJK after surgery for adult spinal deformity. Osteoporos Int. 2016;27:3495-3502. https://doi.org/10.1007/s00198-016-3676-6

\section{Figures}
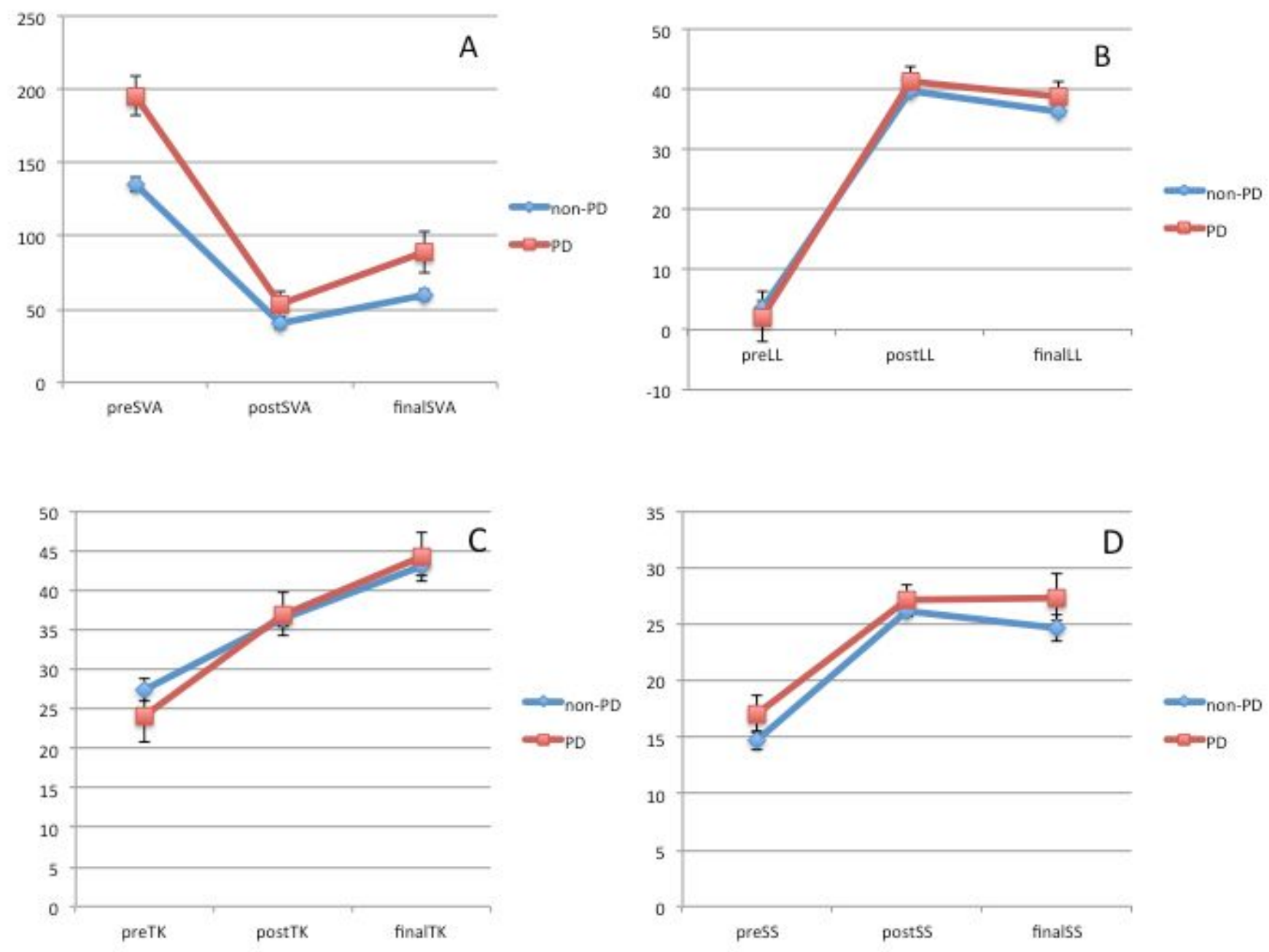

\section{Figure 1}

Comparison of change in radiographic parameters between patients with Parkinson's disease (PD) and those without PD (non-PD) Line graph showing change in mean radiographic parameters preoperatively, 
postoperatively, and at final follow-up. Black bars indicate standard errors. (A) Patients with PD show higher preoperative sagittal vertical axis than those without PD. Both groups show significant correction in sagittal vertical axis postoperatively; patients with PD showed higher correction loss at final follow-up. (B) (C)(D) No significant differences were found preoperatively, postoperatively, and at the final follow-up value for other radiographic parameters, including correction and correction loss. Figure created using Microsoft Office PowerPoint 2011. 